\title{
COMPARACIÓN DE DOS MODELOS EXPERIMENTALES DE GLAUCOMA EN CONEJOS
}

\author{
COMPARISON OF TWO EXPERIMENTAL MODELS OF GLAUCOMA IN RABBITS
}

L. Ignacio Tártara ${ }^{1,2,4}$, Stefania Leavi ${ }^{1}$, Vilma R. Campana ${ }^{1,3}$, Daniel A. Allemandi', Santiago D. Palma².

\section{Resumen:}

Objetivo: comparar dos modelos de glaucoma experimental por inducción de hipertensión ocular en conejos y describir cambios anatomo-patológicos. Materiales y métodos: Se utilizaron 16 conejos New Zealand, hembras, de 2-3 kg. Modelo $A(n=6)$ : cauterización de venas epiesclerales y perilimbares en ojo derecho (OD) con cauterio eléctrico quirúrgico. Modelo $B(n=10)$ : inyección intracamerular en OD de a-quimotripsina. Se midió la presión intraocular (PIO) antes y después de la inducción de la hipertensión ocular (HTO), una vez por semana, a la misma hora del día, durante 40 días, con tonómetro manual. Los animales fueron sacrificados por inhalación de $\mathrm{CO}_{2}$. En ambos modelos la PIO del ojo izquierdo (OI).fue tomado como valor control. La media y error estándar (EE) de los valores de la PIO, expresada en $\mathrm{mmHg}$, fueron evaluadas y comparadas estadísticamente aplicando Test T de Student considerando un nivel de significación de $p<0.05$. Resultados: La PIO en Ol (control) del modelo A: fue 12,9 $\pm 1,05$ y en el modelo B: 12,9 $\pm 1,09$. No se observaron diferencias significativas entre ambas. Modelo A: el aumento de la PIO en OD fue $14,7 \%(14,8 \pm 1,4)$ con respecto a OI. Se observó un incremento significativo de la PIO dentro de las primeras 24 hs: $23,5 \pm 1,9(p<0,05)$ comparado con el valor del ojo control. No hubo diferencias significativas con los controles posteriores. Modelo B: el aumento de la PIO en OD fue $129,1 \%(29,6 \pm 3,4)$ con respecto al OI. En todos los casos se observó un incremento desde el día 1 $(p<0,05)$. El pico de PIO en OD se evidenció el día 25: 35 $\pm 3,4(p<0,05)$. El incremento de la PIO inducida en el modelo $B$ fue significativamente mayor $(p<0,01)$ que en el modelo $A$. En ambos modelos hubo pérdida de células ganglionares de la retina, pero sólo en el modelo $B$ se observaron los siguientes cambios anatomo-patológicos: buftalmus, subluxación del cristalino y aumento de la excavación de la papila. Conclusión: De acuerdo a este estudio, el modelo $B$ aparece como el método más apropiado a los fines de inducir un incremento rápido y controlado de la IOP en conejos y más importante, este incremento sería capaz de mantenerse alto a lo largo de periodos de tiempos extendidos. Este modelo podría ser de gran utilidad para evaluar la eficacia de nuevos sistemas oculares de liberación de fármacos y realizar futuros estudios de la fisiopatología del glaucoma.

Palabras clave: modelo experimental de glaucoma; a-quimotripsina; cauterización de las venas epiesclerales; hipertensión ocular.

\section{Abstract:}

Objective: to compare two models of experimental glaucoma by induction of ocular hypertension in rabbits.

Materials and methods: Sixteen New Zealand female rabbits, 2-3 kg were used. Model A $(n=6)$ : cauterization of episcleral and perilimbar veins of the right eye (RE) with surgical electrocautery. Model $B$ $(n=10)$ : Injection of $\alpha$-chymotrypsin in posterior chamber of RE. Intraocular pressure (IOP) was measured before and after the induction of ocular hypertension (OHT), once a week at the same time of day for 40 days, with a manual tonometer. The animals were euthanized by $\mathrm{CO}_{2}$ inhalation. In both models the control was the IOP of the left eye (LE). The mean and standard error (SE) values of IOP, expressed in $\mathrm{mmHg}$, were compared statistically by applying Student's t-test with a significance level of $p<0.05$. Results: The IOP in LE (control) of model A: was $12.9 \pm 1.05$ and in model B: $12.9 \pm 1.09$. There were no significant differences between the models. Model A: The IOP increase in RE was $14.7 \%(14.8 \pm 1.4)$ with respect to LE. A significant increase in IOP was observed within the first 24 hours: $23.5 \pm 1.9(p<0.05)$ compared to the control eye. There were no significant differences with subsequent controls. Model B:

1 Cátedra de Física Biomédica. Facultad de Ciencias Médicas. UNC

2 Departamento de Farmacia. Facultad de Ciencias Químicas. UNC

3 Cátedra de Física Biomédica. Medicina. UNLaR

4 Email de contacto: i.tartara@gmail.com

Recibido: 2017-04-04 
The increase in IOP in RE was $129.1 \%(29.6 \pm 3.4)$ with respect to LE. In all cases an increase was observed from Day $1(p<0.05)$. The IOP peak in RE was evidenced on Day 25: $35 \pm 3.4(p<0.05)$. The increase in IOP induced by model B was significantly higher $(p<0.01)$ than in model A. There was loss of ganglion cells of the retina in both models, but the following anatomo-pathological changes were observed only in model B: buphthalmos, subluxation of the lens and increased excavation of the papilla. Conclusion This study indicates that model B is the most appropriate method to induce a rapid, controlled increase of IOP in rabbits and, more importantly, that this increase may be sustained over extended periods of time. This model could be useful for evaluating the efficacy of new ocular drug delivery systems and for further studies of the physiopathology of glaucoma.

Keywords: experimental glaucoma model; a-chymotrypsin; cauterization of episcleral veins; ocular hypertension. 


\section{Introducción}

El glaucoma describe un grupo de trastornos oculares de etiología multifactorial cuyo factor común es la neuropatía óptica progresiva ${ }^{1}$. Es un desorden que compromete a las células ganglionares de la retina (CGR), provocando degeneración y muerte de las mismas, pérdida del campo visual y finalmente, la ceguera. Es una de las principales causas de ceguera y discapacidad visual que afectan a la población mundial, se estima que existen 70 millones de personas que padecen esta enfermedad ${ }^{2,3}$.

El factor de riesgo más importante asociado con el glaucoma es el aumento de la presión intraocular (PIO), y el único hasta ahora factible de ser modificado ${ }^{4}$. La muerte de las neuronas de la retina es el punto común final entre el glaucoma y la enfermedad de la retina ${ }^{5}$. Como tal, las terapias hipotensoras y neuroprotectoras son una necesidad terapéutica imprescindible para los pacientes que padecen esta enfermedad ${ }^{6}$.

A pesar de los esfuerzos e investigaciones en el campo del glaucoma, su fisiopatología aun no ha sido totalmente esclarecida.

Los modelos animales han ayudado en gran medida a la comprensión de las causas y la progresión de las enfermedades humanas y han demostrado ser una herramienta útil para descubrir dianas para la terapia farmacológica y quirúrgicas. Sin embargo, varias afecciones permanecen incurables porque no todos los modelos utilizados para el estudio de estas patologías han podido ser reproducidos completamente ${ }^{7}$.

Para estudiar el glaucoma se han utilizado grandes animales como monos ${ }^{8}$, perros ${ }^{9}$, gatos ${ }^{10}$ y $\operatorname{cerdos}^{11} ;$ y otros pequeños, tales como ratas, cobayos y conejos ${ }^{12}$. Se han implementado diferentes técnicas para provocar glaucoma experimental, como por ejemplo el uso de láser, obliteración de venas epiesclerales, inyección de sustancias a nivel intraocular y sistémico, la aplicación de corticoides tópicos y generales, etc ${ }^{13}$.

Como se mencionó anteriormente, los modelos experimentales de glaucoma son esenciales para esclarecer el curso natural de la enfermedad y el desarrollo de intervenciones terapéuticas útiles para detener o revertir la progresión de la patología ${ }^{14}$. El uso de un mamífero como el conejo, permite reproducir un excelente modelo basado en la similitud de su anatomía con la humana, permitiendo realizar procedimientos quirúrgicos semejantes a la clínica oftalmológica. Además de ser un animal de fácil manipulación--presenta condiciones de mantenimiento accesibles y costos relativamente bajos.

Por lo antes mencionado, nos hemos propuesto reproducir y comparar dos modelos de glaucoma por inducción de hipertensión ocular (HTO) en conejos y describir los cambios anatomo-patológicos producidos.

\section{Materiales y métodos}

Se utilizaron 16 conejos albinos tipo New Zealand, hembras, de 2-3 kg. Los conejos recibieron alimento y agua ad libitum en una sala con temperatura controlada $\left(21^{\circ} \pm 5^{\circ} \mathrm{C}\right)$, expuesto a ciclos de 12 hs de luz, 12 hs de oscuridad.

Todos los procedimientos experimentales se ajustaron a la normas ARVO (Association for Research en Visión y Oftalmología) y el Consejo de las Comunidades Europeas Directiva (86/609/CEE) sobre el uso de animales en la investigación. Los protocolos fueron revisados y aprobados por el Comité de Uso Cuidado Institucional de animales de Facultad de Ciencias Químicas de la Universidad Nacional de Córdoba, Córdoba, Argentina. Resolución 342/2009 del HCD de la FCQ. Después de una semana de adaptación en las instalaciones, los animales fueron admitidos a las sesiones experimentales.

Modelo $A(n=6)$ : cauterización de venas epiesclerales ${ }^{15}$ (CVEE) y vasos perilimbares de ojo derecho (OD) con cauterio eléctrico quirúrgico.

Técnica quirúrgica: se realizó peritomía conjuntival 360oㅡㄹ se localizaron 3 venas epiesclerales y se cauterizaron con electrocauterio portátil bipolar punta fina (HTCF726600). Luego se realizaron aproximadamente 15 quemaduras perilimbares con el mismo cauterio, se cerró la conjuntiva con nylon 9.0. Se aplicó antibiótico y antiinflamatorio no esteroideo tópico durante 7 días.

Modelo $B(n=10)$ : inyección intracamerular en OD de a-quimotripsina ${ }^{16}$.

Técnica quirúrgica: se instiló pilocarpina al $2 \%$ para provocar miosis, luego se realizó una paracentesis corneal superior y se inyectó en cámara posterior con cánula de 27G 0,1 ml de a-quimotripsina (C425$250 \mathrm{mg}$ laboratorio Sigma), en una concentración de $3 \mathrm{mg} / \mathrm{ml}$. Al cabo de 3 minutos se lavó la cámara anterior con solución fisiológica estéril. Se aplicó antibiótico y antiinflamatorio no esteroideo tópico durante 7 días. Al ojo izquierdo (OI) utilizado como control, se le inyectó $0,1 \mathrm{ml}$ de solución fisiológica estéril en cámara posterior. 
En ambos modelos se midió la PIO antes y después de la inducción de la HTO, cada 5 días durante 40 días con tonómetro manual (Icare TonoVet. Icare USA) debidamente calibrado. También se realizó fotografía digital del segmento anterior y fondo de ojo con máquina fotográfica adosada a la lámpara de hendidura (Huvitz HIS 5000. Corea del Sur). A todos los animales se les realizó anestesia general con xilacina $(0,2 \mathrm{ml} / \mathrm{kg})$ y ketamina $(0,8 \mathrm{ml} / \mathrm{kg})$. En ambos modelos el control fue la PIO del Ol expresada en $\mathrm{mmHg}$. Al completar el examen los animales fueron sacrificados por inhalación de $\mathrm{CO}_{2}$, previa sedación con xilacina $(0,2 \mathrm{ml} / \mathrm{kg})$. Ex vivo se enuclearon ambos ojos, se realizaron preparados histológicos con tinción de hematoxilina \& eosina y se procedió al análisis descriptivo comparativo de las retinas (capa de células ganglionares) entre los ojos hipertensos y normales, mediante microscopía óptica (microscopio óptico Olympus BX41. Japón)

Análisis estadístico: Los resultados fueron analizados aplicando T. de Student (tiempos individuales) y MANOVA ( $\mathrm{T}$. de Hotelling) para comparar la PIO en ambos modelos, considerando un nivel de significación de $p<0.05$.

\section{Resultados}

La PIO en OI (control) del modelo A: fue 12,9 $\pm 1,05$ y en el modelo $B: 12,9 \pm 1,09$. Sin existir diferencias significativas entre ambas.

Modelo A (ver Fig. 1): el aumento de la PIO en OD fue $14,7 \%(14,8 \pm 1,4)$ con respecto al OI. Se observó un incremento significativo de la PIO dentro de las primeras 24 hs: $23,5 \pm 1,9(p<0,05)$ comparado con el ojo control. No hubo diferencias significativas en los controles posteriores.

Modelo B (ver Fig. 1): el aumento de la PIO en OD fue $129,1 \%(29,6 \pm 3,4)$ con respecto al OI. En todos los casos se midió un incremento desde el 1ํ día $(p<0,05)$. El pico de PIO en OD se evidenció el 25ํ día: $35 \pm 3,4(p<0,05)$.

El incremento de la PIO inducida por el modelo $B(29,6 \pm 3,4)$ fue significativamente mayor $(p<0,01)$ que en el modelo $A(14,8 \pm 1,4)$.

En el modelo $A$ se constató una disminución leve a moderada de la cantidad de CGR (fig 2 b) con respecto al preparado de retina normal (fig 2 a). Mientras que en el modelo $B$ se observó una marcada disminución de CGR. (fig 2 c). Sólo en el modelo $B$ se observaron cambios anatomo-patológicos: buftalmus (fig. $3 a$ y b), subluxación del cristalino (fig. $3 c$ y d) y aumento de la excavación de la papila (fig. $3 \mathrm{f})$.

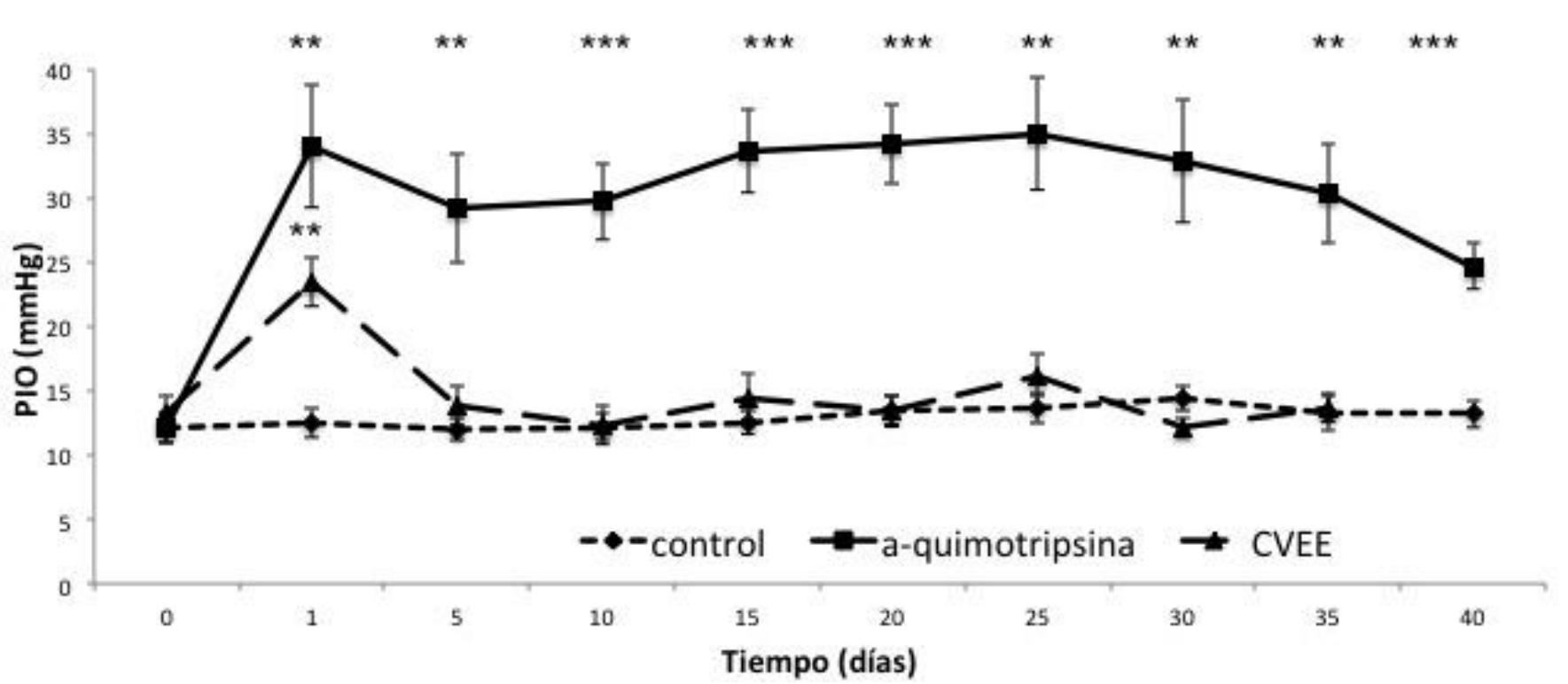

Figura 1. Comparación de PIO en ojos de conejos normales y PIO de ojos con el Modelo A y B. Línea punteada representa la PIO de los ojos controles ( $\mathrm{n}=16$ ), línea discontinua representan la PIO de los ojos tratados con Modelo A (CVEE), ( $n=6)$. Por último la línea continua indica la PIO del Modelo B ( $\mathrm{n}=10)$. Cada par ordenado representa la media \pm EE de los valores de $\mathrm{PIO}$ en todos los tiempos expresada en $\mathrm{mmHg} .{ }^{* *} \mathrm{p}<0,01{ }^{* * *} \mathrm{p}<0,001$. 


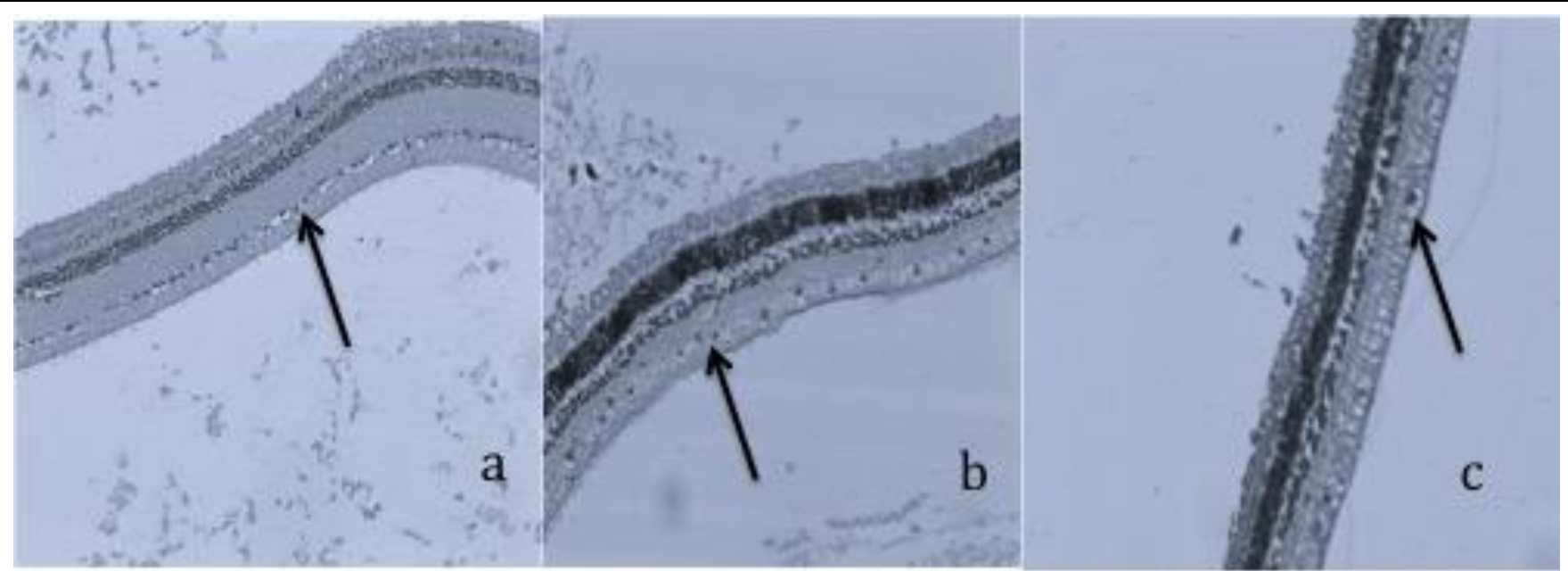

Figura 2. Fotografía de cortes histológicos de retina de ojos de conejos. H\&E, 10X. Fotografía a) Ojo normotenso; b) Ojo de modelo A; c) Ojo de modelo B. Las flechas indican la capa de células ganglionares

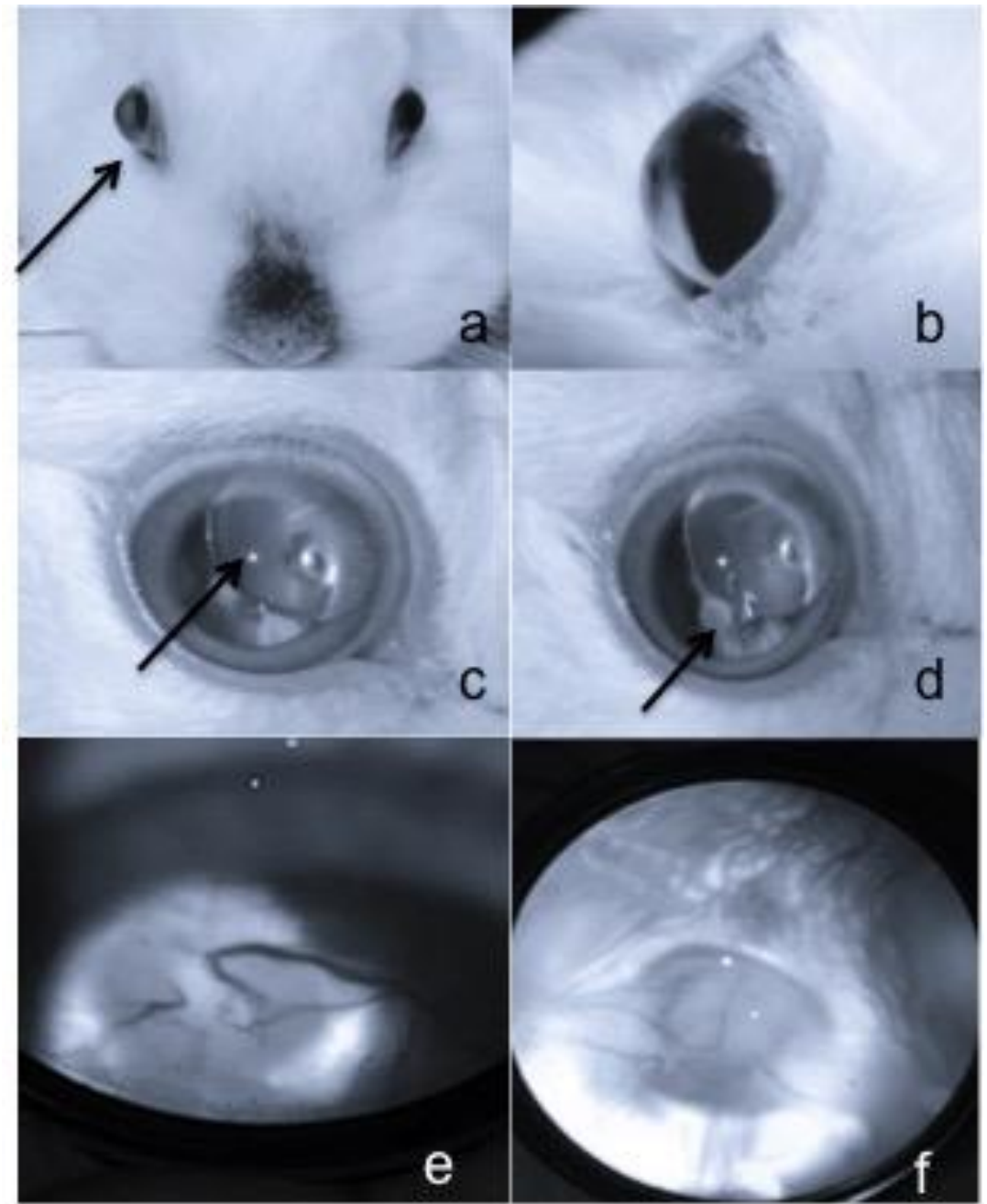

Figura 3. a- fotografía de conejo (modelo B), se observa el agrandamiento del OD (flecha) producto de la HTO (buftalmus). En la fotografía -b- se observa en detalle el crecimiento del global ocular, abombamiento de la córnea y aumento del espacio en la cámara anterior del ojo. Fotografía -c- se observa la subluxación superior del cristalino la flecha señala el cristalino, en - $d$ - la flecha muestra por retroiluminación el reflejo rojo de la retina. En -e- se muestra una fotografía biomicroscópica de nervio óptico normal de ojo de conejo. En -f- Fotografía del nervio óptico realizada a los 20 días de la inyección de a-quimotripsina con excavación aumentada (modelo B).

\section{Discusión}

El glaucoma es una enfermedad compleja con una fisiopatología que está lejos de ser completamente comprendida. Es probable que el desarrollo continuo de modelos animales, y su exploración en profundidad de la patología, contribuyan en el futuro al esclarecimiento de aspectos relacionados con esta enfermedad y su terapia en pacientes humanos. Los modelos en roedores son particularmente 
ventajosos debido a su alto grado de disponibilidad, el costo relativamente bajo, corta duración de vida y la facilidad para la manipulación experimental y genética ${ }^{17}$. Sin embargo, dichos modelos no son reproducciones exactas de la condición humana. La anatomía del ojo del conejo es bastante similar al humano, y además posee la ventaja de ser un animal de fácil manipulación y costos relativamente accesibles. Por tal motivo se lo ha seleccionado para la aplicación en glaucoma experimental.

La CVEE es una técnica que se ha utilizado pretendiendo elevar la PIO en ratas mediante la cauterización quirúrgica de dos o más venas epiesclerales, con ello se afecta el drenaje del humor acuoso $^{18}$.

En nuestro trabajo de investigación utilizamos este modelo con una modificación que consiste en la cauterización de los vasos perilimbares en conejos (modelo A). Esto permitió generar un aumento de la $\mathrm{PIO}$ del $15 \%$ en todos los conejos, aunque dicho incremento tuvo una duración de solo 24 horas. Nuestra experiencia encontró marcadas diferencias con los resultados de Ruiz-Ederra y Verkman ${ }^{15}$, quienes al realizar esta técnica en ratones, lograron un aumento de la PIO de alrededor del $100 \%$ y que persistió durante 4 semanas. Probablemente, la diferencia de especies animales utilizadas no permita la comparación del mecanismo de mantenimiento de la HTO. Dicho mecanismo es explicado por Morrison y col $^{19}$ quienes sugieren que se ralentiza el drenaje del humor acuoso en las unidades organizativas de los túbulos colectores del canal de Schlemm con un aumento de la congestión venosa ocular. En este modelo, Garcia-Valenzuela y $\mathrm{col}^{20}$, determinaron que el número de venas epiesclerales cauterizadas se correlaciona con el grado de elevación de la PIO, la cual produce una pérdida esperada de CGR a una velocidad de aproximadamente $4 \%$ por semana. Además, se demostró que la muerte de las CGR inducida por la HTO, se lleva a cabo al menos en parte, a través de la apoptosis. En nuestro caso, cauterizamos tres venas epiesclerales, ya que cuando se intentó cauterizar más venas, las complicaciones postquirúrgicas como hipema y edema corneal, se produjeron en todos los casos, conejos que debieron ser descartados para el presente estudio. En cuanto a las CGR, los análisis descriptivos de los preparados histológicos (fig 4 b), demostraron una pérdida de las mismas, pero no se pudo concluir ni la velocidad, ni el porcentaje de pérdida, ya que el período de HTO fue muy corto: 24 hs. Otra técnica empleada para aumentar la PIO en modelos animales es mediante la inyección de $\alpha$ quimotripsina en la cámara posterior del $0_{j}{ }^{21,22}$. Si bien no es un procedimiento utilizado actualmente para inducir glaucoma, la utilización de este modelo en nuestro caso demostró diferentes resultados a los hallados en el modelo A. El aumento de la PIO fue de aproximadamente un $130 \%$, estable y con un pico de $35 \mathrm{mmHg}$ a las 3 semanas de la inducción. Resultados similares fueron publicados por Best y $\mathrm{col}^{16}$ en ojos de conejos mediante la inyección de 75 unidades de a-quimotripsina en la cámara posterior, donde la elevación de la PIO rara vez excedió los $50 \mathrm{mmHg}$ y duró un año o más. Vareilles y $\mathrm{Col}^{23}$, reportaron hallazgos similares a los encontrados en nuestra investigación, tales como: buftalmus progresivo que aparecieron en las primeras 2 a 3 semanas después de la inyección de la enzima; cambios histológicos oculares que incluyen queratopatía bullosa, la atrofia del iris y del cuerpo ciliar y la excavación de la papila óptica. En la retina hubo adelgazamiento de la capa de fibras nerviosas y la pérdida de células ganglionares con la preservación de los otros elementos de la retina. En experimentación también observamos subluxación del cristalino.

El mecanismo que conduce a glaucoma después de la inyección de a- quimotripsina no está claro. Probablemente la inflamación que provoca la enzima conduce a sinequias como las descriptas por Best y $\mathrm{col}^{16}$ bloqueando la salida de humor acuoso a través del trabeculado. Chee y $\mathrm{col}^{24}$ determinaron que el bloqueo de la malla trabecular en monos Rhesus era debido a la lisis del material zonular. Lessell y $\mathrm{col}^{25}$ postulan una atrofia del cuerpo ciliar.

El modelo $A$, si bien permitió generar aumento de la PIO en conejos, no se mantuvo en el tiempo y los valores fueron significativamente menores que en el modelo $B$. Además, el primer modelo requiere una destreza quirúrgica especializada, mayor tiempo en la realización de la técnica y una curva de aprendizaje más empinada. Con respecto a los cambios anátomo-patológicos observados en el examen oftalmológico del conejo, el modelo $B$ presentó en todos los casos buftalmus (fig $3 a$ y b) que es el agrandamiento del globo ocular producto de la HTO; subluxación del cristalino (fig $3 \mathrm{c}$ y d) por lisis de fibras zonulares, producto de la acción de la enzima y el crecimiento del diámetro ocular; midriasis paralítica, signo indirecto de ceguera; aumento considerable de la excavación papilar (fig 3 e y f) lo que demuestra la pérdida de fibras nerviosas de la retina; y en los preparados histológicos: una marcada pérdida de CGR (fig 2 c). Todos estos cambios no se observaron en el modelo $A$, a excepción de la leve a moderada pérdida de CGR (fig 2 b); estas diferencias se debieron al poco tiempo de HTO que experimentaron los conejos del modelo $A$. 


\section{Conclusión}

De acuerdo a este estudio, el modelo B aparece como el método más apropiado a los fines de inducir un incremento rápido y controlado de la $\mathrm{PIO}$ en conejos y más importante, este incremento sería capaz de mantenerse alto a lo largo de periodos de tiempos extendidos. Este modelo podría ser de gran utilidad para evaluar la eficacia de nuevos sistemas oculares de liberación de fármacos y realizar futuros estudios de la fisiopatología del glaucoma.

\section{Agradecimientos}

Trabajo financiado por: SECyT-UNC. Res. 313/16. Proyecto: Plataformas nanotecnológicas de liberación de fármacos (PNLFs) para el tratamiento de patologías oculares - PICT-2012-1358

\section{Bibliografía}

1. Casson RJ, Chidlow G, Wood JP, Crowston JG, Goldberg I. Definition of glaucoma: clinical and experimental concepts. Clin Exp Ophthalmol 2012; 40(4): 341-9.

2. Quigley H, Broman AT. The number of people with glaucoma worldwide in 2010 and 2020 . Br J of Ophthalmol 2006; 90(3): 262-7.

3. Thylefors B, N'egrel AD, Pararajasegaram R, Dadzie KY. Available data on blindness (update 1994). Ophthalmic Epidemiol 1995; 2(1): 5-39.

4. The AGIS Investigators. The advanced glaucoma intervention study (AGIS) VII. The relationship between control of intraocular pressure and visual field deterioration. A J Ophthalmol. 2000; 130(4): 429-40,

5. Garcia-Campos J, Villena A, Diaz F, Vidal L, Moreno M, Perez de Vargas I. Morphological and functional changes in experimental ocular hypertension and role of neuroprotective drugs. Histol Histopathol 2007; 22(12): 1399-1411.

6. Bagnis A, Papadia M, Scotto R, Traverso CE. Current and emerging medical therapies in the treatment of glaucoma. Expert Opin Emerg Drugs 2011; 16(2): 293-307.

7. Vecino E. Animal models in the study of the glaucoma: past, present and future. ArchSocEspOftalmol 2008; 83(9): 517-19.

8. Rasmussen CA, Kaufman PL. Primate glaucoma models. J Glaucoma 2005; 14(4): 311-14.

9. Brooks DE. Glaucoma in the dog and cat. Vet Clin North Am Small Anim Prac 1990; 20(3):775-97.

10. Dietrich U. Feline glaucomas. Clin Tech Small An P 2005; 20(2):108-16.

11. Ruiz-Ederra J, García M, Hernández M, et al. The pig eye as a novel model of glaucoma. Exp Eye Res 2005; 81(5):561-69.

12.Pang IH, Clark AF. Rodent models for glaucoma retinopathy and optic neuropathy. J Glaucoma 2007; 16(5): 483-505.

13. Zeiss CJ. Translational models of ocular disease. Vet Ophthalmol 2013; 16(1): 15-33.

14. Agarwal R, Agarwal P. Rodent models of glaucoma and their applicability for drug discovery. Expert Opin Drug Discov 2017; 12(3): 261-270.

15. Ruiz-Ederra J, Verkman AS. Mouse model of sustained elevation in intraocular pressure produced by episcleral vein occlusion. Exp Eye Res 2006; 82(5): 879-84.

16. Best M, Rabinovitz AZ, Masket S. "Experimental a-chymotrypsin glaucoma". Ann Ophthalmol. 1975; 7(6): 80310.

17. Yu S, Tanabe T, Yoshimura N. A rat model of glaucoma induced by episcleral vein ligation. Exp Eye Res 2006; 83(4): 758-70.

18. Shareef SR, Garcia-Valenzuela E, Salierno A, Walsh J, Sharma SC. Chronic ocular hypertension following episcleral venous occlusion in rats. Exp Eye Res 1995; 61(3): 379-82.

19. Morrison JC, Moore CG, Deppmeier LMH, Gold BG, Meshul CK, Johnson EC. A rat model of chronic pressureinduced optic nerve damage. Exp Eye Res 1997; 64(1): 85-96.

20.Garcia-Valenzuela E, Shareef S, Walsh J, Sharma SC. Program med cell death of retinal ganglion cells during experimental glaucoma. Exp Eye Res. 1995; 61(1): 33-44.

21. Zhu MD, Cai FY. Development of experimental chronic intraocular hypertension in the rabbit. Aust $N Z J$ Ophthalmol.1992; 20(3): 225-34.

22. Melena J, Santafe J, Segarra J. The effect of topical dihydroergocristine on the intraocular pressure in alphachymotrypsin-induced ocular hypertensive rabbits. Methods Find Exp Clin Pharmacol 1998. 20(10): 861-867.

23. Vareilles P, Durand G, Siou G, Le Douarec JC. Experimental alpha-chymotrypsin model of glaucoma in the rabbit: histopathological studies. J Fr Ophtalmol. 1979; 2(10): 561-8.

24. Chee P, Hamasaki DI. The Basis for Chymotrypsin-Induced Glaucoma. Arch Ophthalmol 1971; 85(1): 103-06.

25. Lessell S, Kuwabara T. Experimental alpha-chymotrypsin glaucoma. Arch Ophthalmol 1969; 81(6): 853-64. 